\title{
SAGITTALIZATION OF THE OBLIQUE MUSCLES AS A POSSIBLE CAUSE FOR THE "A", "V", AND "X" PHENOMENA* ${ }^{\dagger}$
}

\author{
BY \\ M. H. GOBIN \\ From the University Eye Hospital of Leyden, Netherlands \\ (Director: Prof. M. C. Colenbrander, M.D.)
}

IN order to reduce the incomitance of the " $V$ " phenomenon we carry out an anteroposition of the inferior oblique muscle (Gobin, 1964). This means that the scleral insertion of the muscle is displaced towards the equator, thus enlarging the angle between the muscle and the visual axis. This operation changes the relationship between the torsional and vertical components of the muscle, favouring the torsional action. Our good results suggest that an abnormal relationship between those two components may be an obstacle to binocular vision.

Our concept of the aetiology of the "A", "V", and "X" phenomena is derived from this consideration; before we explain the theory we will first mention their main characteristics.

\section{Clinical Characteristics}

The "A", "V", and "X" phenomena show definite horizontal and vertical incomitance: horizontal when the eyes move vertically and vertical when the eyes move horizontally. It is the horizontal variation of the angle of squint which accounts for the terms " $A$ ", " $V$ ", and " $\mathrm{X}$ ".

(1) "A" Phenomenon

HORIZONTAL INCOMITANCE: The angle of deviation is more convergent on looking up than on looking down.

Vertical InCOMItANCE: The adducted eye is lower than the abducted eye. This is

(2) " $V$ " Phenomenon called a depression in adduction.

HoRIzONTAL INCOMITANCE: The angle of deviation is more convergent in depression than in elevation.

Vertical Incomitance: The adducted eye is higher than the abducted eye. This is

(3) " $X$ " Phenomenon called an elevation in adduction.

Horizontal InCOMITANCE: The angle of deviation is more convergent in the primary position than on looking up or down.

VerTICAL INCOMITANCE: On looking up there is elevation in adduction, and on looking

(4) Asymmetrical Types down there is depression in adduction.

In asymmetrical " $X$ " phenomena the incomitance is unequal in elevation and depression. In the " $\mathrm{X}_{\mathrm{A}}$ " phenomenon the relative divergence is LESS on looking up than on looking down, and in the " $\mathrm{Xv}$ " phenomenon the relative divergence is GREATER on looking up than on looking down.

* Read before the "Boerhaave" Course, Leyden, September 4 and 5, 1964 . Received for publiçation October 14, 1966,

$\dagger$ Address for reprints: Gounod Street 1, Antwerp, Belgium. 


\section{Pathogenesis}

Each vertical muscle has a horizontal, vertical, and torsional action. The mutual relationship between these three components depends upon the angle between the muscle and the visual axis. This angle varies individually especially in relation to the obliques.

Fink (1962) made an extensive study of this subject. In 122 human specimens, he measured the relation of the planes of action of the two oblique muscles to one another, and their relation to the antero-posterior vertical plane of the eyeball. He determined the average angle formed by the oblique muscles with the antero-posterior vertical plane; this was $44.9^{\circ}$ for the inferior oblique and $45.5^{\circ}$ for the superior oblique. However, of those 122 specimens, 22 differed considerably from the others. The greatest deviations were:

When the superior oblique shows the greater angle: superior oblique $64^{\circ}$, inferior oblique $33^{\circ}$.

When the inferior oblique shows the greater angle: superior oblique $32^{\circ}$ and inferior oblique $57^{\circ}$.

When the muscles run nearly parallel, the maximum angle was $67^{\circ}$ for the superior and $60^{\circ}$ for the inferior oblique, and the minimum $22^{\circ}$ for the superior and $34^{\circ}$ for the inferior oblique.

Fink concludes: "A study of individual specimens revealed in many instances an appreciable variation in the muscle plane of one oblique as compared with the other. They varied sufficiently from each other to raise a question as to a balanced action between the two muscles."

These marked variations are not so surprising if one considers that, unlike the rectus muscles, the obliques have a distinct origin, and that the bones to which they are attached, the frontal bone for the superior oblique and the maxilla for the inferior oblique, are phylogenetically different. This may certainly lead to some divergence in their development influencing their mutual relationship. In addition, the scleral insertion undergoes a radical shift during the phylogenetical evolution, being progressively displaced towards the posterior pole of the eyeball. Every asymmetry in this displacement may also be expected to influence the relation between the two obliques, and this altered relation may influence the torsional balance of the eye.

We feel that the cause of the " $A$ " and "V" phenomena may be a break-down of the parallelism between the obliques, whereas the cause of the " $\mathrm{X}$ " phenomenon may be a torsional imbalance between the obliques and the vertical rectus muscles. The cyclophoria which thus arises can be neutralized by an adaptation of the ratio of contraction of the vertical muscles. But this may disturb the balance between the horizontal and vertical components of these muscles, resulting in horizontal and vertical incomitance.

(I) " $A$ " Phenomenon.-We presume that the " $\mathrm{A}$ " incomitance is due to a torsional imbalance between the two oblique muscles. This is due to a reduction of the angle between the superior oblique and the visual axis. This we term a sagittalization of the muscle; it increases the vertical and reduces the torsional action, leading to excyclophoria. This sagittalization occurs when the bulbar insertion of the superior oblique lies further posterior, or when the trochlea is anterior to the corresponding attachments of the inferior oblique (Fig. 1).
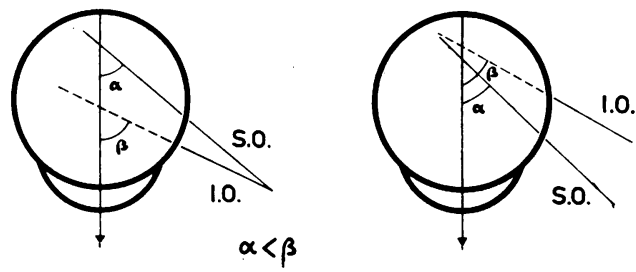

FIG, 1,-Sagittalization of the superior oblique. 


$$
\text { “ } A \text { ", “ } V \text { ", AND "X" PHENOMENA }
$$

The excyclophoria can be compensated for by a contraction of the intorsional muscles and an inhibition of the extorsional muscles, i.e. a contraction of the superior rectus and superior oblique and inhibition of the inferior rectus and inferior oblique. This change in contraction of the vertical muscles produces a depression in adduction and an " $A$ " variation of the horizontal angle of squint (Figs 2 and 3). This " $A$ " incomitance is thus secondary to the removal of the excyclophoria. It should not be underestimated, because it is the result of the co-operation of all the vertical muscles. The size of the horizontal and vertical incomitance and their mutual relationship varies, as do the horizontal and vertical components of these muscles.
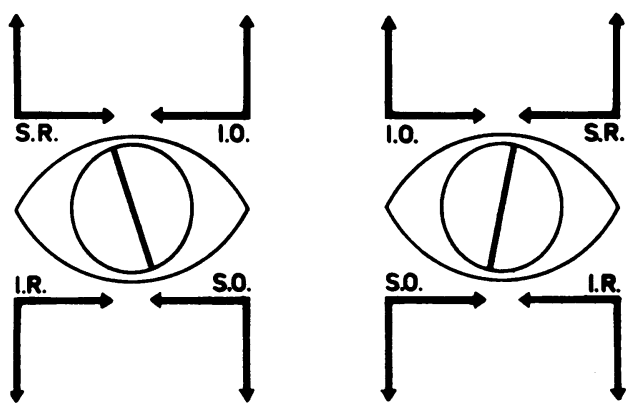

FIG. 2.- "A" incomitance, caused by excyclophoria due to sagittalization of the superior oblique.

The arrows are drawn in the vertical field of action of the muscles. They are drawn of equal length to indicate that the muscles are in vertical and horizontal balance with each other It is not intended to imply, for example, that the vertica action of the superior oblique is equal in strength to that of the inferior rectus.

The vertical and horizontal components of these muscles balance each other. However, the decreased torsional action of the sagittalized superior oblique causes excyclophoria.
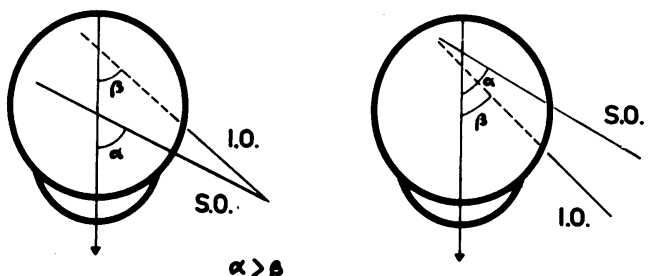

FIG. 4.-Sagittalization of the inferior oblique muscle.

(2) " $V$ " Phenomenon.-We presume that the "V" incomitance is also due to a lack of torsional balance between the obliques, caused in this case by a sagittalization of the inferior oblique. The angle between this muscle and the visual axis is reduced, and this results in a decreased extorsional and an increased vertical action, leading to incyclophoria.

This sagittalization occurs when the bulbar insertion of the inferior oblique lies further posterior, or when its origin is anterior to the corresponding insertions of the superior oblique (Fig. 4).

The incyclophoria of the eyes can be compensated for by a contraction of the extorsional muscles and an inhibition of the intorsional muscles, i.e. a contraction of the inferior rectus and inferior oblique and inhibition of the superior rectus and superior oblique. This change in contraction of the vertical muscles produces an elevation in adduction and a " $V$ " variation of the horizontal angle of squint (Figs 5 and 6, overleaf). 

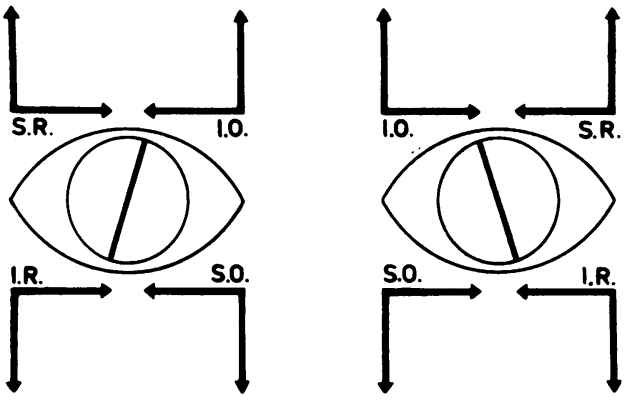

FIG. 5. - "V" incomitance, caused by incyclophoria due to sagittalization of the inferior oblique.

The vertical and horizontal components of the vertical muscles balance each other. The incyclophoria arises because of the sagittalization of the inferior oblique.
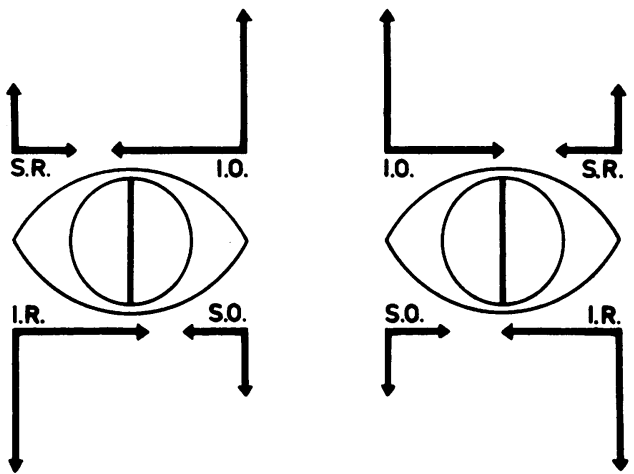

FIG. 6.- "V" incomitance, leading to "V" variation and elevation in adduction.

This incyclophoria can be reduced by contraction of the extorsional muscles and inhibition of the intorsional muscles. This, however, alters the vertical and horizontal effect of these muscles, so that elevation in adduction and " $V$ " variation occurs.

(3) " $X$ " Phenomenon.-We presume that the " $X$ " incomitance is due to a torsional imbalance between the obliques on the one hand and the vertical rectus muscles on the other. Both obliques have too small a torsional action as compared with their horizontal and vertical action so that they do not balance the vertical rectus muscles. This may be due to a sagittalization of both the obliques, whereby their torsional action is decreased and their vertical action increased (Fig. 7), leading to incyclophoria on looking up and excyclophoria on looking down.
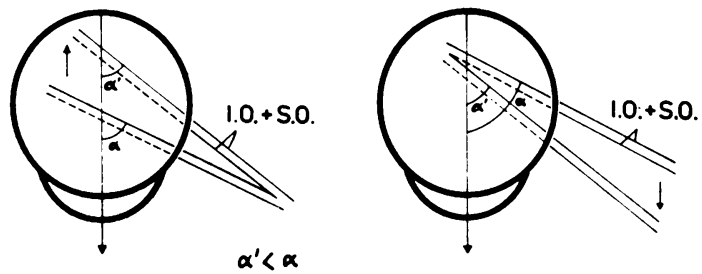

Fig. 7.-Sagittalization of both oblique muscles.

On looking up the extorsional muscles will be contracted and the intorsional muscles inhibited, and on looking down the intorsional muscles will be contracted and the extorsional muscles inhibited; i.e. on elevation there is contraction of the inferior oblique and inhibition of the superior rectus, and on depression there is contraction of the superior oblique and inhibition of the inferior rectus. This leads to a vertical and horizontal preponderance of the obliques over the vertical rectus muscles. The vertical preponderance causes an elevation in adduction on looking up and a depression in adduction on looking down. The horizontal preponderance gives rise to the " $X$ " variation of the angle of squint (Figs 8 and 9, opposite).

(4) Asymmetrical Types.-When the two oblique muscles run parallel we find a symmetrical " $X$ ". When they make an angle with each other we find an " $X_{\mathrm{A}}$ " type (i.e. the superior oblique shows a greater sagittalization than the inferior oblique) and an " $\mathrm{Xv}$ " type is seen in the reverse case.

The varying positions of the oblique muscles may lead to cyclophoria. The optomotor torsional reflexes can compensate for this cyclophoria by varying the contraction of the 

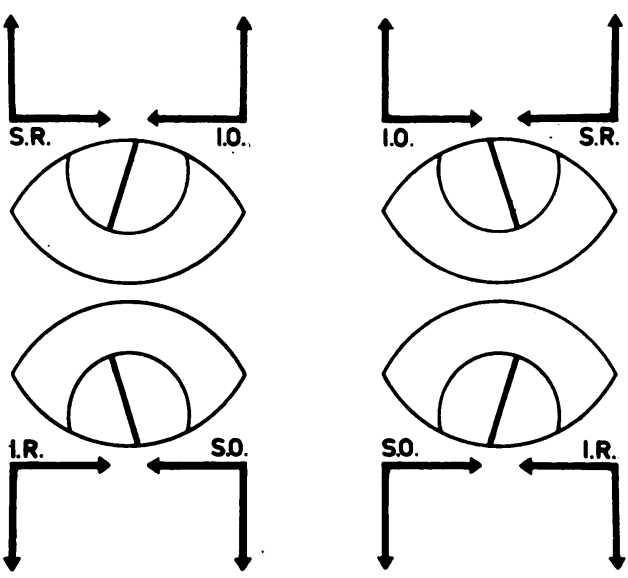

FIG. 8.- "X" incomitance, caused by incyclophoria on looking up and excyclophoria on looking down.

When the vertical muscles balance each other vertically and horizontally, the eyes show incyclophoria on looking up and excyclophoria on looking down.
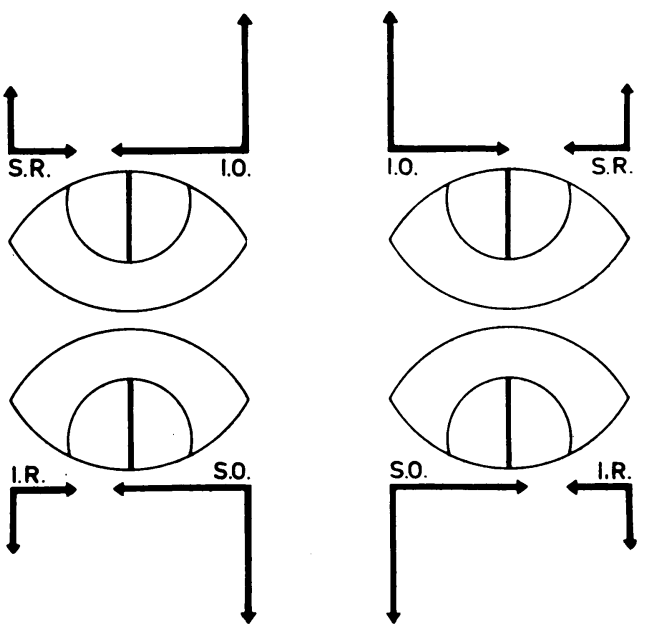

FIG. 9.- " $\mathrm{X}$ " incomitance, leading to the "V" phenomenon on looking up and the " $A$ " phenomenon on looking down.

The incyclophoria on looking up can be reduced by a contraction of the inferior oblique and an inhibition of the superior rectus. This, however, increases the vertical and horizontal actions of the inferior oblique and decreases those of superior rectus and thus gives rise to an elevation in adduction and to a tendency to divergence.

The excyclophoria on looking down can be reduced by a contraction of the superior oblique and an inhibition of the inferior rectus which leads to a depression in adduction and again to a tendency to divergence.

vertical muscles. If, however, the horizontal and vertical balance between these muscles is broken by this adaptation, horizontal or vertical incomitance will arise, causing a conflict between the torsional balance of the eyes and the concomitance of the eye movements. Although both are necessary for binocular vision, a certain amount of cyclophoria is acceptable, because it allows bifoveal fixation. Evidence for this can be found in the large torsional fusion range which is demonstrable on the synoptophore. This appears to indicate that the meridians of the eye do actually show an inclination at certain times and/or in certain positions of gaze.

Under the influence of the fusion reflexes, the incomitance is kept latent at the cost of a certain degree of cyclophoria. But either alone or in combination with other factors this cyclophoria can make binocular vision impossible. The diplopia which appears when binocular vision is interrupted leads to suppression, and this in turn eliminates the fusional reflexes. Thus the incomitance is no longer kept in check and gradually becomes manifest.

If the fusional reflexes are able to compensate for the incomitance once the causative factors are removed, the original cyclophoria will be restored. If, however, the fusional reflexes are not able to do this (when the suppression is too dense or when the incomitance has become too pronounced) cyclophoria will be absent.

The good results of our surgery of the oblique muscles in cases of " $V$ " phenomenon can readily be understood, because the operation corrects the angle between the muscle and the visual axis.

\section{Summary}

The "A", "V", and " $X$ " phenomena may be caused by cyclophoria. The incomitance of the eye movements may be produced by variation in the contraction of the vertical muscles, which tends to remove the torsional imbalance of the eye. This cyclophoria is 
thought to be caused by a too sagittal course of the superior oblique in the " $A$ " phenomenon, of the inferior oblique in the " $V$ " phenomenon, and of both obliques in the " $X$ " phenomenon.

\section{BIBLIOGRAPHY}

AdLer, F. H. (1948). Amer. J. Ophthal., 31, 387. (1953). A.M.A. Arch. Ophthal., 50, 19.

BURIAN, H. M. (1944). Amer. J. Ophthal., 27, 1284.

Crone, R. A. (1952). “Alternende Hyperphorie", pp. 50-113. Swets and Zeitlinger, Amsterdam, Netherlands.

Duane, A. (1932). Arch. Ophthal. (Chicago), 8, 530.

Dunnington, J. H. (1931). Amer. J. Ophthal., 14, 1140.

FinK, W. H. (1962). "Surgery of the Vertical Muscles of the Eye", 2nd ed., pp. 196-202. Thomas, Springfield, Ill.

FolK, E. R. (1965). "Treatment of Strabismus". Thomas, Springfield, Ill.

GoBIN, M. H. (1964). Ophthalmologica (Basel), 148, 325.

HugonNIER, R. (1958); Bull. Soc. Ophtal. Fr., p. 585. (1962). Ibid., p. 286.

JAMPOLSKY, A. (1957). Trans. Amer. Acad. Ophthal. Otolaryng., $61,689$.

KrewsON, W. E. (1950). Ibid., 48, 443.

Noto, K. (1961). Jap. J. Ophthal., 5, 261.

RUBINSTEIN, K., and Dixon, J. (1959). Brit. J. Ophthal., 43, 21.

SACHSENWEGER, R., and LuKOFF, L. (1959). Klin. Mbl. Augenheilk., 135, 388.

Urrets-Zavalia, A. (1961). Trans. Amer. Acad. Ophthal. Otolaryng., 65, 324.

URIST, M. J. (1951). A.M.A. Arch. Ophthal., 46, 245. (1958). Amer. J. Ophthal., 46, 835.

VillasECA, A. (1961). Ibid., 52, 172.

WAGMAN, O. H. (1945). I Ibid., 28, 1226.

Weiss, J. B. (1964). Bull. Soc. Ophtal. Fr., p. 251.

ZAKI, H. A. A., and KeENEY, A. H. (1957). A.M.A. Arch. Ophthal., 57, 418. 